\title{
Dielectric Analysis of Response Time in Electrorheological Fluids Developed for Medical Devices
}

\author{
Naullage Indika Perera, Manik Pavan Maheswaram, Dhruthiman Mantheni, \\ Hettiarachchige Dhanuja Perera, Michael Ellen Matthews, Tobili Sam-Yellowe, Alan Riga \\ Department of Chemistry, Cleveland State University, Cleveland, USA \\ E-mail:alanriga@hotmail.com
}

Received August 2, 2010; revised January 12, 2011; accepted March 5, 2011

\begin{abstract}
Three electrorheological fluids (ERFs) of recently synthesized Polyaniline. $\mathrm{HCl}$ and Cellulose fluids as well as a commercial product from Fludicon ${ }^{\circledR}$ (Germany), were evaluated with a two-electrode probe unit and by Dielectric Analysis (DEA). The study was a part of an ongoing medical device development project. The dielectric response times were calculated using the critical peak frequency in a corresponding Debye plot of Tan Delta (loss factor/permittivity) vs. log frequency. The DEA revealed the response times (tau, $\tau$ ) in ms. The Fludicon ${ }^{\circledR}$ ERF was DEA durable (repeat cycles produced same results) and the $\tau$ was temperature dependent: $16 \mathrm{~ms}$ at $25^{\circ} \mathrm{C}$ and $0.16 \mathrm{~ms}$ at $80^{\circ} \mathrm{C}$. The Cellulose ERF was somewhat DEA durable and the $\tau$ was $5.5 \mathrm{~ms}$ at $25^{\circ} \mathrm{C}$ and $0.21 \mathrm{~ms}$ at $80^{\circ} \mathrm{C}$. The respo nse times were logarithmic with the temperature (C) wi th a correlation coefficient of $>0.98$ for the Cellulose and Fludicon ${ }^{\circledR}$ ERFs. The Polyaniline ERF had a $\tau$ of 53 $\mathrm{ms}$ at $25^{\circ} \mathrm{C}$ in the $1^{\text {st }} \mathrm{DEA}$ run and there was no indication of a $\tau$ for the remaining DEA tests.
\end{abstract}

Keywords: ERF, Tan Delta, DEA, Fibrillation, Debye Plot, Response Time

\section{Introduction and Background}

Electrorheological fluids (ERFs) are generally suspensions of high dielectric constant electrically polarizable particles in non-conducting base fluids with a low dielectric constant. ER active materials are generally polymers, ceramics, metals or composites such as cellulose, starch, titanium oxide, polyurethanes and polyanilines [1-5]. In the presence of an external AC electric field, typically a few kilovolts per millimeter, these ER active particles are polarized due to the dielectric difference between the suspended particles and the base fluid [6]. The field-induced dipoles on the particles cause them to align and form chains or fibrillated structures that bridge the electrode gap. The formation of chains or fibrillation result in a large and reversible increase in the apparent viscosity (see Figure 1) that can be easily adjusted by controlling the strength of the applied electric field.

Ever since the discovery of the ER concept by Willis Winslow (1949) in the late 1930s [7], a tremendous amount of research on various applications of ERFs has been going on in university and industrial labs due to their potential use as rapid, infinitely variable interfaces between electrical and mechanical components of intel- ligent systems [8-11]. ERFs are now used in many industrial applications, i.e. hydraulic valves, clutches, brakes, and shock absorbers. Although utilization of ER technology in current medical device development applications is low, the interest has been growing in recent years. Less agglomeration, low power consumption, better dispersability and faster response, favorable in milliseconds, to an applied field are all important factors for
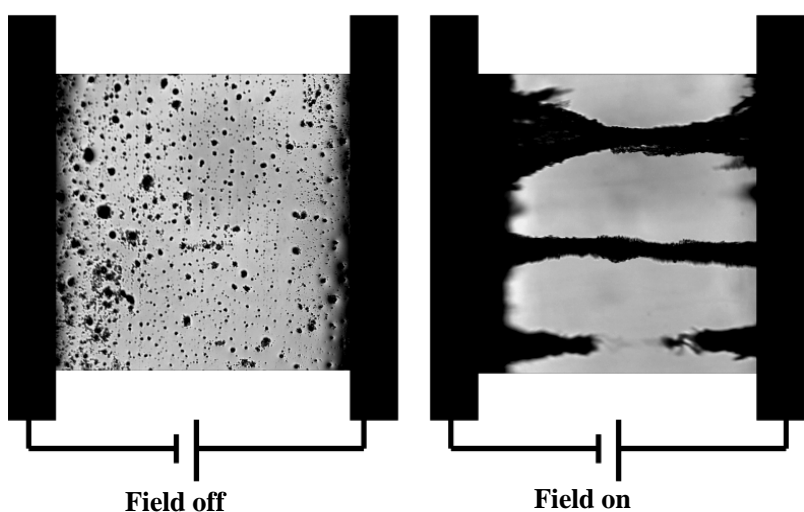

Figure 1. Particle fibrillation of Fludicon ${ }^{\circledR}$ RheOil 3.0 ERF. Imaged at Riga imaging facility. 
an ideal ERF. The results of the ideal behavior are being considered for use in device development applications. Of all the above mentioned ERF qualities, the response time is a significant parameter that should be considered for many applications when selecting a suitable ERF. Therefore, accurate measurement of ERF response time is crucial. There are many methods currently available in the market that can be employed to measure ER response time effectively and accurately, the DEA method developed by Riga et al. is promising in terms of experimental time [12], cost and repeatability. DEA is a widely used thermal analytical method that measures polarization response in an AC electric field at isothermal temperatures or by scanning temperature techniques [13-16]. A Debye plot of Tan Delta, a ratio of dielectric loss divided by the relative permittivity, versus frequency can fix the limits of ER active particle polarization or relaxation time. The ER response time in a commercial ERF is directly related to the polarization time, which is inversely related to the critical peak frequency in the Debye plot.

In the present work, we analyzed DEA response times in three widely used ERFs and their suitability for using in medical device development applications. The ERFs used in this study are Polyaniline Hydrochloride (PANI$\mathrm{HCl}$ ), Cellulose and Fludicon ${ }^{\circledR}$ RheOil 3.0 from the Fludicon ${ }^{\circledR}$ Cooperation, Germany. PANI-HCl ERFs were selected in this study mainly due to their favorable stability, adjustable conductivity, controllable particle size, low density and hardness, which are all good qualities for many applications. Cellulose ERFs exhibit most of the above mentioned properties in addition to relatively low cost and ease of synthesis. The key advantage of including Fludicon ${ }^{\circledR}$ RheOil 3.0, which contains polyurethane particles doped with $\mathrm{Zn}^{2+}$ in silicone oil, in this study is that it is a commercialized product and has already been used in device development applications $[17,18]$.

\section{Experimental Procedure}

\subsection{Chemicals and Commercial ERFs}

All materials needed for ERF synthesis, Aniline (Cat\# 242284), Hydrochloric Acid (Cat\# H1758), Microgranular Cellulose (Cat\# C6413), Ethylene Glycol (Cat\# 324558), Ammonium Persulfate (Cat\# A3678), 5 cSt Silicone Oil (Cat \#317667), SPAN 85 surfactant were reagent grade and purchased from Sigma-Aldrich. Deionized water was obtained from a Barnstead ultra pure water purification system (specific resistance $>18.2 \mathrm{M} \Omega / \mathrm{cm}$ ). Fludicon ${ }^{\circledR}$ RheOil 3.0 was purchased from Fludicon ${ }^{\circledR}$ Cooperation in Germany.

\subsection{Procedures and Apparatus}

\subsubsection{Preparation of Cellulose ERF}

Cellulose ERFs were prepared using a standard protocol. Briefly, cellulose polymers were heated in an oven overnight at $120^{\circ} \mathrm{C}$. The dried cellulose was then placed in a desiccator until it came to room temperature. Next, 30\% dried cellulose, 3.0\% anhydrous ethylene glycol, 2\% SPAN $85^{\circledR}$ were ball milled overnight in 5 cSt silicone oil. The final ERF was collected in a glass container and left in a desiccator until used.

\subsubsection{Preparation of Polyaniline ERF}

Synthesis and Purification of Polyaniline-HCl (PANI$\mathrm{HCl}) 500 \mathrm{~mL}$ of $1 \mathrm{M}$ Aniline in $1 \mathrm{M} \mathrm{HCl}$ was mixed with $500 \mathrm{~mL}$ of $1 \mathrm{M}$ Ammonium Persulfate in a beaker at $0^{\circ} \mathrm{C}$. The reaction mixture was gently stirred and left at rest to polymerize overnight. Next day, the PANI precipitate was collected on a filter paper, washed three times with $0.1 \mathrm{M} \mathrm{NH}_{4} \mathrm{OH}$, and similarly with deionized water. The resultant PANI. $\mathrm{HCl}$ was dried in an oven at $120^{\circ} \mathrm{C}$ for 48 hours, and then placed in a desiccator until needed. PANI. HCl structure was confirmed using IR spectroscopy.

\subsection{Preparation of the PANI. HCI ERF}

$\mathrm{pH}$ of the previously synthesized PANI-HCl was adjusted to a desired $\mathrm{pH}$ (e.g. 7.0) by doping and de-doping with $\mathrm{NH}_{4} \mathrm{OH}$ and $\mathrm{HCl}$. After doping/de-doping process, the PANI. HCl was collected on a filter paper, washed three times with deionized water, and similarly with Methanol. Next, the $\mathrm{pH}$ adjusted PANI. $\mathrm{HCl}$ was dried in an oven for 48 hours at $120^{\circ} \mathrm{C}$.

The ER fluid was prepared by dispersing the PANI. HCl in silicone oil in the presence of the SPAN 85 surfactant. The final ER fluid was obtained by ball milling PANI, SPAN 85 and 5 cSt silicone oil for 4 hours. The composition of the final ERF was 15\% wt PANI, 3\% wt SPAN 85 in 5 cSt silicone oil.

\subsubsection{Testing ER Fluids for ER Activity}

ER effects and leakage currents of all the ER fluids used in the study were measured at room temperature using an Electrorheological high field probe (to $3.0 \mathrm{kV} / \mathrm{mm}$ ) connected to a voltage amplifier, a RheCon Fludicon ${ }^{\circledR}$ fluid digital control system. The distance between the two electrodes in the probe was set to $1.0 \mathrm{~mm}$. We have designed a novel protocol to measure ER activities in this study and it is measured as follows. First, the high voltage probe is dipped carefully into the ER fluid to a depth of $38 \mathrm{~mm}$ (1.5 inches) (see Figure 2). Next, the electrodes are charged with the variable electric field. The 


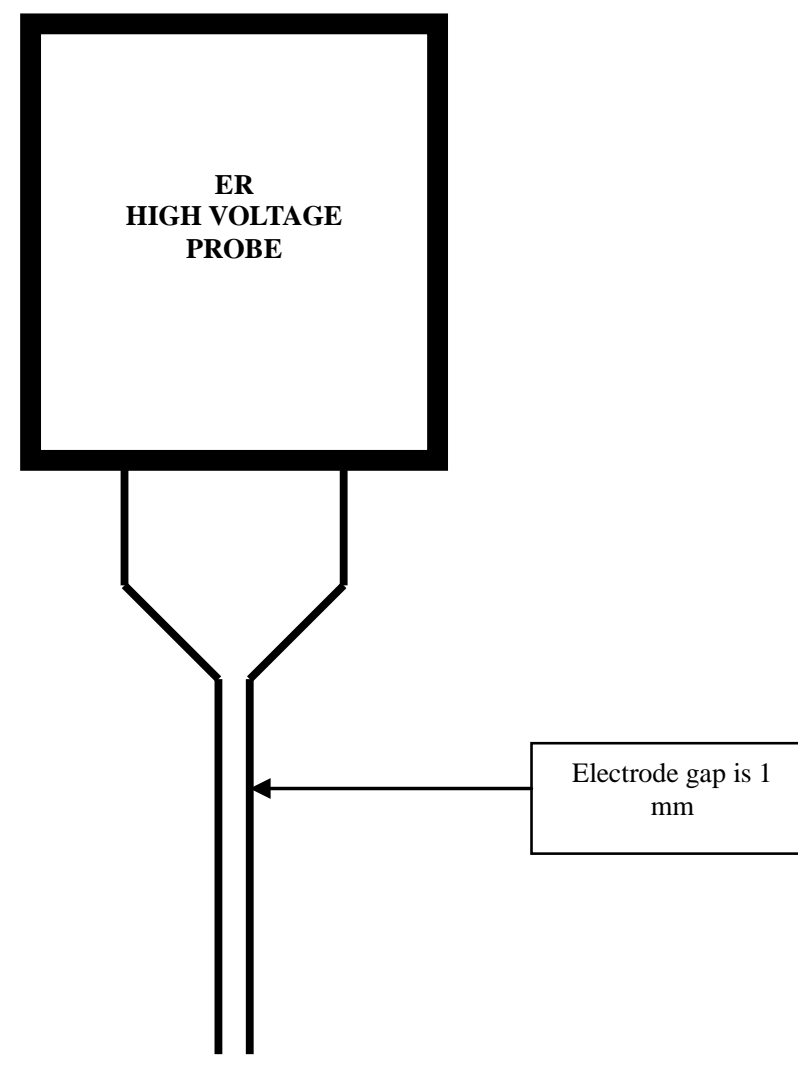

Without Field

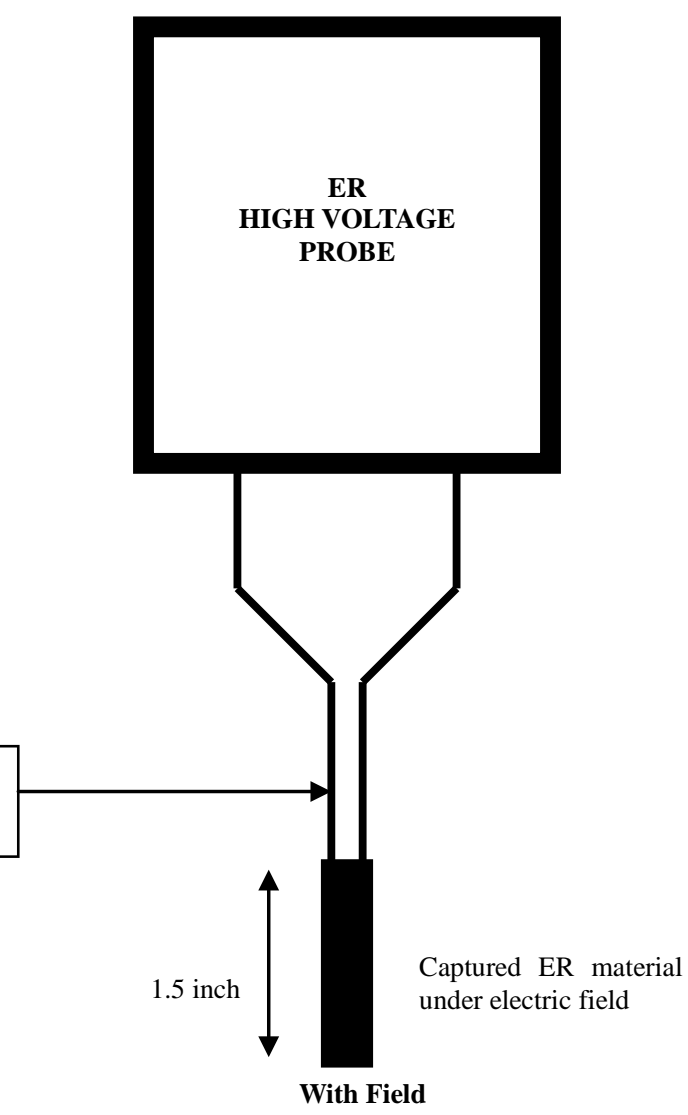

Figure 2. Testing of ERFs using electrorheological high voltage probe.

probe is then taken out and left undisturbed for one minute without releasing the field to allow all loosely bound ER materials to fall off. When the dripping stops, after one minute, the applied electric filed is removed and the ER materials captured between two electrodes are collected and weighed. This process was performed for each ER fluid under varying applied electric fields from 0.5 to $3.0 \mathrm{kV} / \mathrm{mm}$. The captured weights (g) for each ER fluid were plotted against the applied fields.

\subsubsection{Optical Imaging of ER Particles}

Imaging of ER particles was performed using Amscope ${ }^{\circledR}$ microscope digital camera with 5.0 megapixel resolution connected to an American Optical ${ }^{\circledR}$ benchtop microscope. All the imaging was done at $100 \mathrm{X}$ magnification. Dynamic light scattering technique was used to obtain particle dimension information.

\subsubsection{DEA Response Time Analysis of the ERFs}

A TAI 2970 Dielectric Analyzer (TA Instrument) was used to measure the dielectric properties of our ERF systems. The properties studied by DEA are electrical conductivity, permittivity, and tan delta. Gold ceramic single surface interdigitated sensors were calibrated by the in- strument, and were used to evaluate the electrical properties of the ERF systems. Electrical response times were calculated by plotting tan delta vs. log frequency. The DEA applied voltage was $50 \mathrm{~V} / \mathrm{mm}$ and the frequency scan ranged from $0.1 \mathrm{~Hz}$ to $10000 \mathrm{~Hz}$. A drop of solution ( $\sim 50 \mathrm{mg})$ was placed on a sensor. In the first run, samples were isothermally scanned at room temperature for 60 minutes using nitrogen as purge gas at a flow rate of $60 \mathrm{~mL} / \mathrm{min}$. Then the sample is heated from room temperature to $80^{\circ} \mathrm{C}$ at $10^{\circ} \mathrm{C} / \mathrm{min}$ and isothermal for 60 minutes at the test temperature for the second run. For the third run the sample is isothermally scanned at room temperature for $60 \mathrm{~min}$.

\section{Results and Discussion}

\subsection{Testing ER Activities and Measuring Leakage Currents of the ERFs}

All ERFs were tested using the high field probe (0.5 to $3.0 \mathrm{kV} / \mathrm{mm}$ ) connected to a voltage amplifier, RheCon Fludicon ${ }^{\circledR}$ fluid digital control system. The ER activity was measured as described in the experimental section. An ideal ERF changes its apparent viscosity linearly with 
the applied field. Therefore, as a result of this linear behavior, we assume that the amount of captured ER materials using our protocol is linearly proportional to the external field. The leakage currents are also recorded directly from the instrument and this will give another insight into the study as it is desirable to have the strongest ER effect with the lowest leakage current for many applications related to medical device development.

Figure 3 shows the plot of the captured material weight in grams versus applied electric field ( $\mathrm{E}, \mathrm{kV} / \mathrm{mm}$ ) in $\mathrm{kV} / \mathrm{mm}$ for RheOil 3.0 from Fludicon ${ }^{\circledR}$. As predicted, the captured weight increases linearly with the external field indicating an ideally behaved ERF in RheOil 3.0. Also, leakage current values are steady at $0.9 \mu \mathrm{A}$ from 0.5 to $3 \mathrm{kV} / \mathrm{mm}$ (see Figure 3 inset plot). These promising results clearly justify why RheOil 3.0 has been widely used in many applications. Figure 4 presents the captured weight as a function of applied field for the cellulose ERF. The cellulose ERF also behaves similarly to the RheOil 3.0 yielding a slightly low leakage current of $0.8 \mu \mathrm{A}$ (see Figure 4 inset plot). However, our previous studies revealed that activated cellulose particles in their ERFs settles much faster ( $<2 \mathrm{hrs}$ ) compared to $\mathrm{Zn}^{2+}$ doped polyurethane particles in RheOil 3.0 ( $>$ a week). This might be the reason for sparing use of cellulose ERFs in many recent Electrorheological applications. Figure 5 represents captured weight against applied field plot for PANI. HCl ERF. Although it shows a perfect linearity as in cellulose and RheOil 3.0 ERFs, the PANI. $\mathrm{HCl}$ ERFs are relatively unstable in terms of leakage currents, especially after $1.5 \mathrm{kV} / \mathrm{mm}$ (see Figure 5 inset plot).

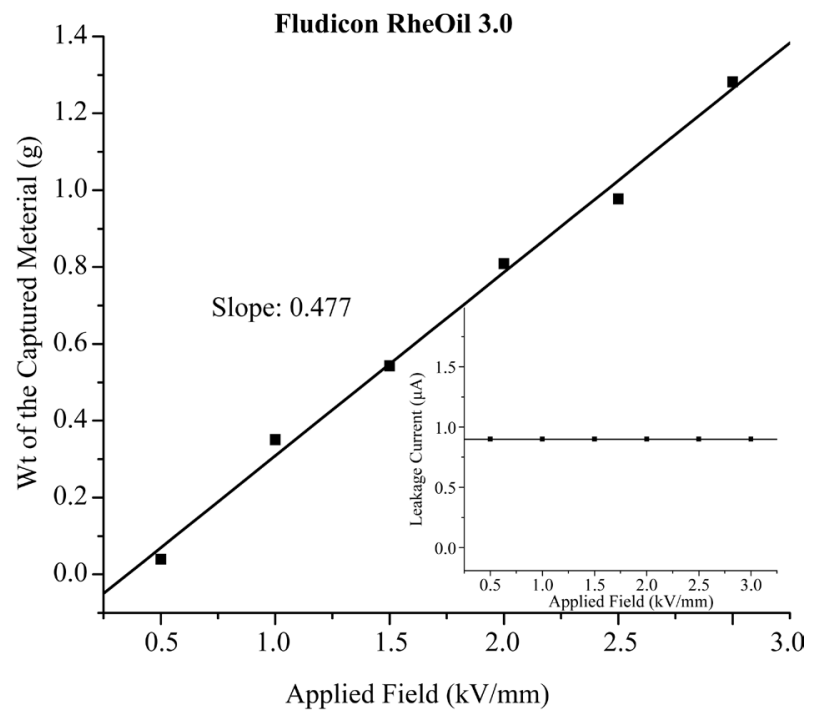

Figure 3. Captured wt. against applied field plot for Fludicon ${ }^{\circledR}$ RheOil 3.0, inset plot shows leakage current values.

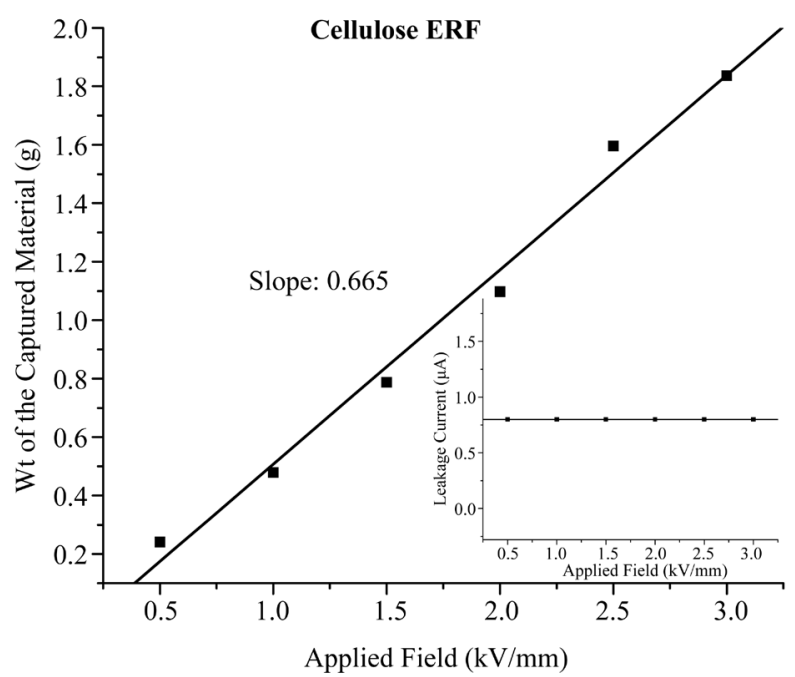

Figure 4. Captured wt. against applied field plot for Cellulose ERF, inset plot shows leakage current values.

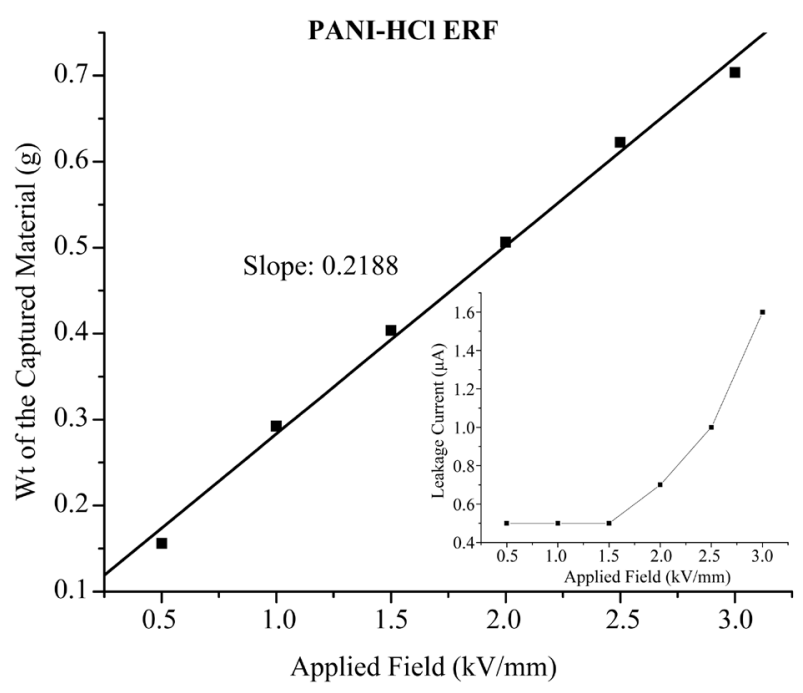

Figure 5. Captured wt. against applied field plot for Polyaniline. HCI ERF, inset plot shows leakage current values.

Interpretation of the RheCon data of captured ERF weight vs. the applied electric field can also be understood by analyzing an ER Bingham plastic model. In this model the ER properties of shear stress $(\sigma, \mathrm{kPa})$ vs shear rate $(\gamma, 1 / \mathrm{s})$ are linear with an increasing applied electric field $(\mathrm{E})$. The response $(\sigma / \gamma)$ is linearly enhanced by the field (E). In a recent publication, a higher ER strength of the fibrillation with field for the Fludicon ${ }^{\circledR}$ ERF was reported by Gurka et al. [19]. Therefore, it is our view that the RheCon slope increases with the strength of the ER effect, a higher slope value can be expected for stronger ERFs. In Figure 6 there is a comparison of the three ERFs studied by the RheCon instrument and the curve slopes are: cellulose, 0.66; Fludicon ${ }^{\circledR}$ RheOil 3.0, 
0.48; and PANI. $\mathrm{HCl}, 0.22$. It is our interpretation that the order of ER strengths evaluated by this high field technique are in the same ranking cellulose $>$ RheOil $3.0>$ PANI. HCl.

\subsection{Optical Imaging of the ERFs}

Figure 7 shows optical imaging of the ERFs. The cellulose particles were significantly larger than either the PANI. $\mathrm{HCl}$ or the commercial Fludicon ${ }^{\circledR}$ RheOil 3.0 particles. The cellulose was on average 27 microns and the PANI. $\mathrm{HCl}$ was 4.0 microns and the Fludicon ${ }^{\circledR}$ was 3.7 microns. Cellulose settles much faster ( $<2$ hours) than either of the other ERFs. This cellulose particle characteristic is a detriment to selecting it as a candidate ERF for a medical device. The settling properties of the other two candidates are minor and the average settling time is $>$ one week.

\subsection{DEA Response Time Analysis of the ERFs}

The key ER property of response time (ms) was determined by DEA. A sample is placed on an interdigitated gold array single surface ceramic electrode and an AC voltage is applied. The DEA applied electric field causes polarization and oscillation of the molecules in the sample at the applied frequencies with a phase angle shift $\Theta$. DEA measures polarization response in an AC electric field at an isothermal temperature. A Debye plot, tan delta vs. log frequency, graphically describe the system polarization time, which is related to molecular mobility. The peak frequency, fc, is inversely related to molecular polarization at a given temperature. Polarization time can be calculated from the following equation:

$T=\left(1 / 2 \Pi f_{c}\right)(1000) m s$ at a given temperature, where $\mathrm{fc}_{\mathrm{c}}$ is the peak frequency in $\mathrm{Hz}$.

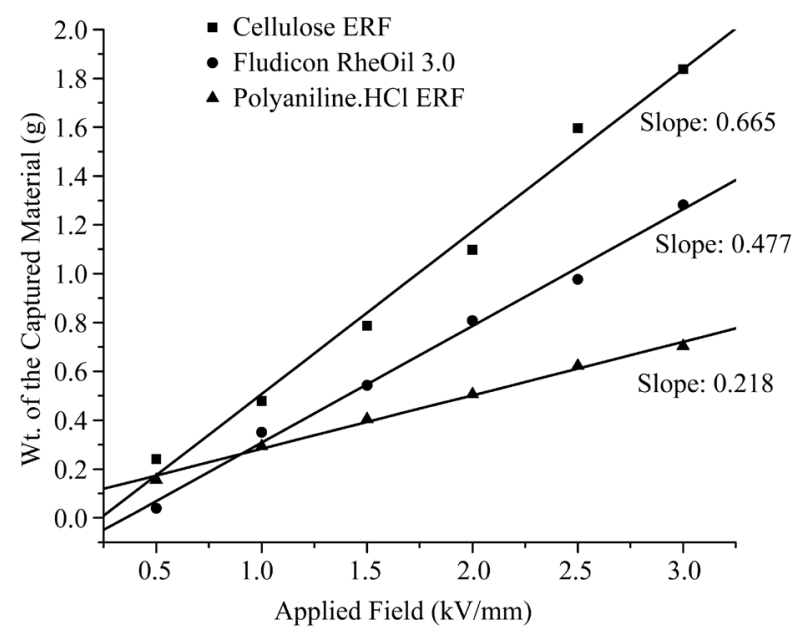

Figure 6. ERF RheCon slope comparison.

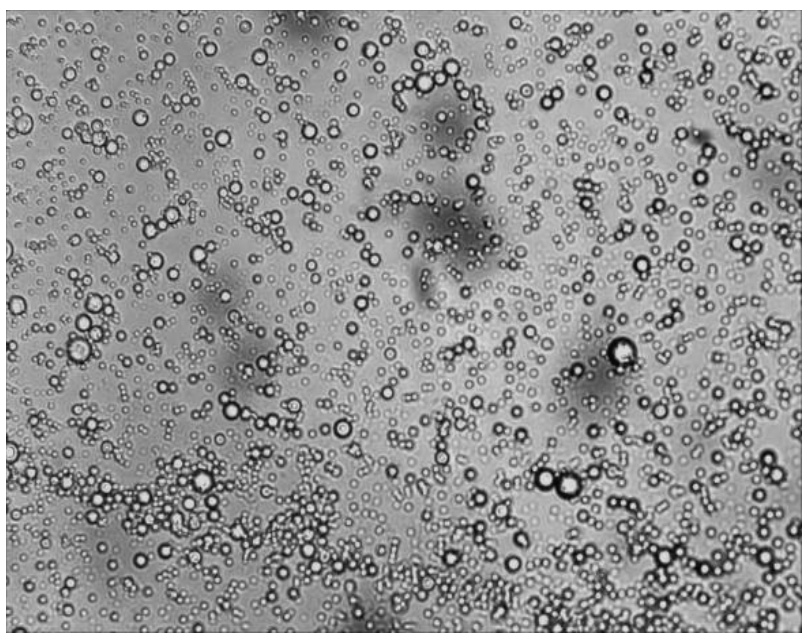

(a)

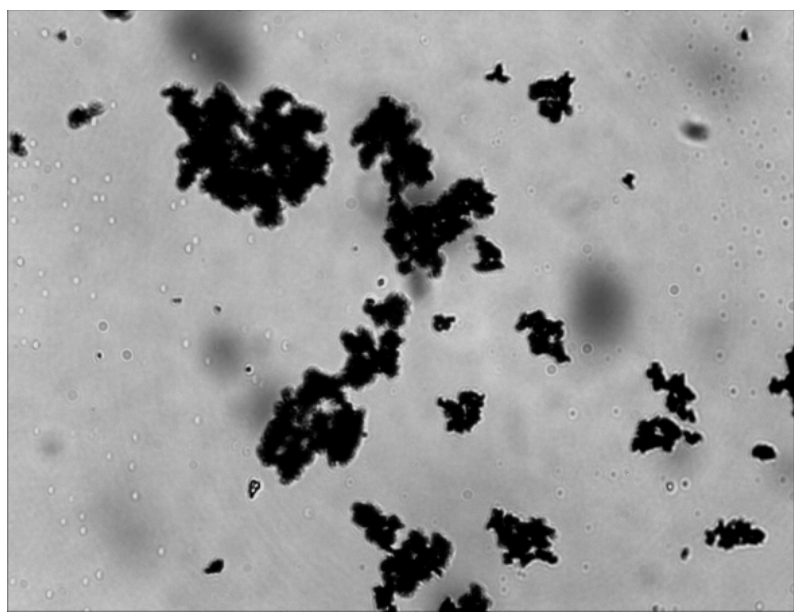

(b)

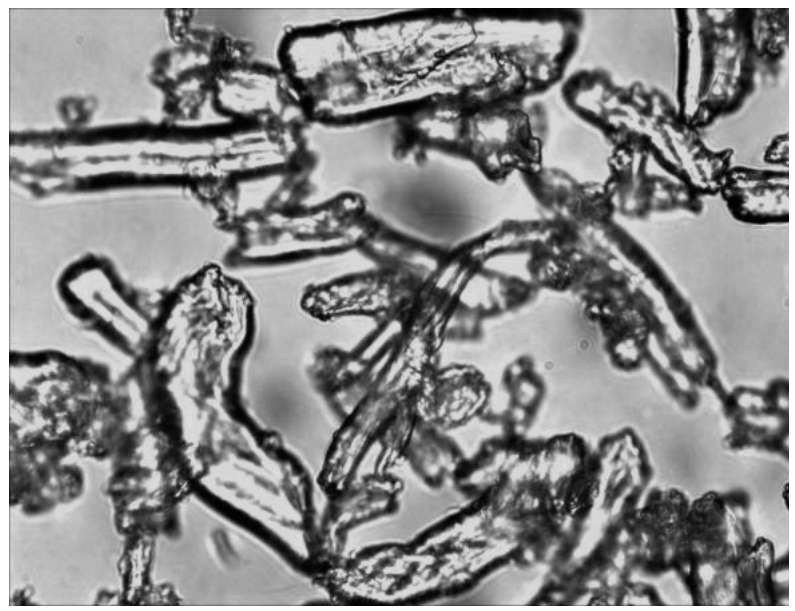

(c)

Figure 7. Optical imaging of ERFs at X100 magnification. (a) Fludicon ${ }^{\circledR}$ RheOil 3.0 ERF; (b) Polyaniline. HCI ERF; (c) Cellulose ERF.

Each ER fluid sample was measured in one hour iso- 
thermal sequences of 25, 80 and $25^{\circ} \mathrm{C}$ (for a total of 3 hours). We consider ERFs to be response time durable if the first and third runs are similar within $1 \mathrm{~ms}$. Figure 8 shows DEA curves of tan delta versus frequency for the Fludicon ${ }^{\circledR}$ RheOil 3.0 ERF. The calculated response time $(\tau)$ for the Fludicon ${ }^{\circledR}$ ERF was $15 \mathrm{~ms}$ at $25^{\circ} \mathrm{C}$ in the first run and $0.16 \mathrm{~ms}$ in the second run at $80^{\circ} \mathrm{C}$. However, in the third run again at $25^{\circ} \mathrm{C}$ a similar response time (16 ms) to the first one was reported. This data clearly indicates the Fludicon ${ }^{\circledR}$ RheOil 3.0 is a DEA durable ERF and the response time is strongly influenced by the temperature. Also, the obtained DEA response times at $25^{\circ} \mathrm{C}$ were very close to the published response time of
$12 \mathrm{~ms}$ at $25^{\circ} \mathrm{C}$ [20]. The Cellulose ERF shows somewhat durability giving $5.5 \mathrm{~ms}$ at $25^{\circ} \mathrm{C}$ in the first run and 1.6 $\mathrm{ms}$ in the third run. Settling in the ERF may be the cause for the lack of reproducibility for the Cellulose ERF. As seen in the Fludicon ${ }^{\circledR}$ ERF, the response times were strongly temperature dependant for the Cellulose ERF giving $0.21 \mathrm{~ms}$ at $80^{\circ} \mathrm{C}$ (see Figure 9). The response times were logarithmic with the temperature $\left({ }^{\circ} \mathrm{C}\right)$ with a correlation coefficient of $>0.98$ for the Cellulose and Fludicon ${ }^{\circledR}$ ERFs. The Polyaniline.HCl ERF had a relatively slow response of $53 \mathrm{~ms}$ at $25^{\circ} \mathrm{C}$ in the first DEA run and there was no indication of a $\tau$ for the remaining DEA tests (see Figure 10).

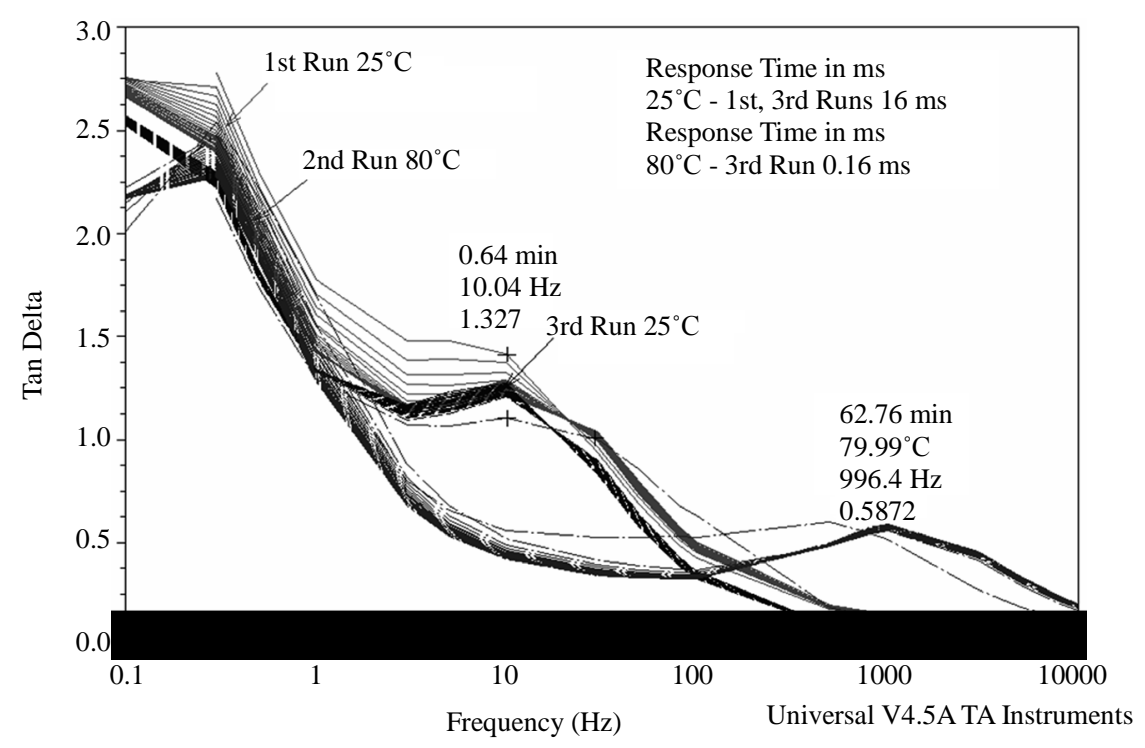

Figure 8. DEA response time analysis for Fludicon ${ }^{\circledR}$ RheOil 3.0 ERF.

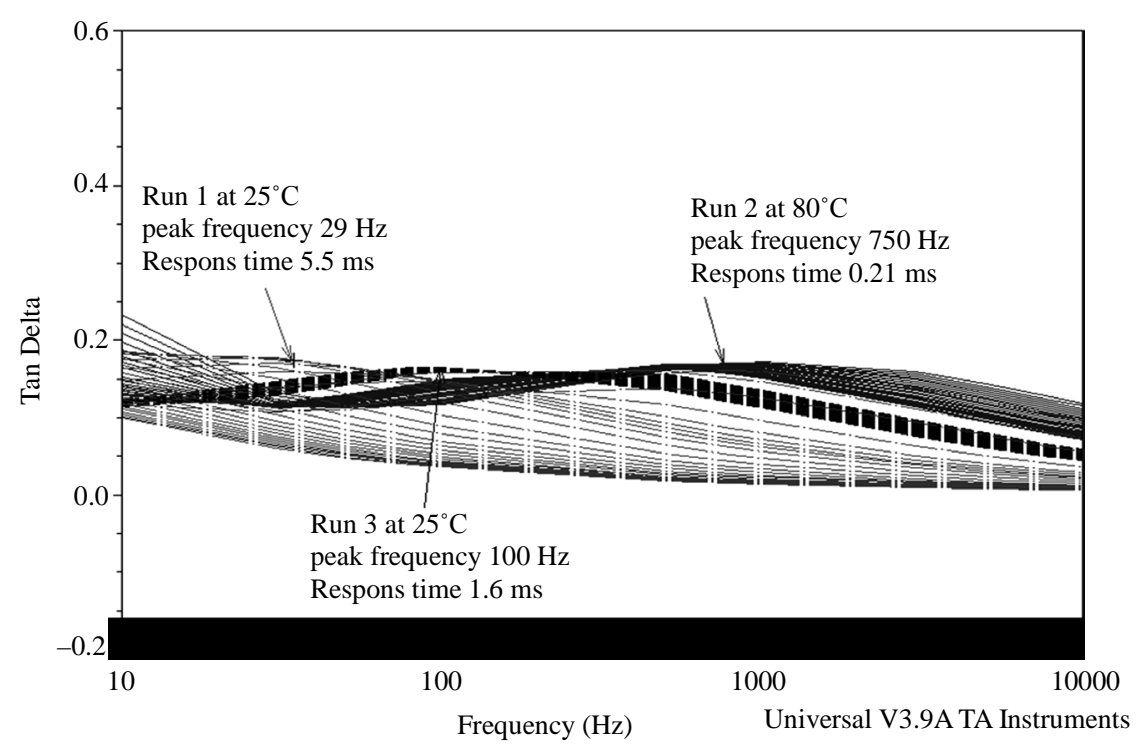

Figure 9. DEA response time analysis for Cellulose ERF. 


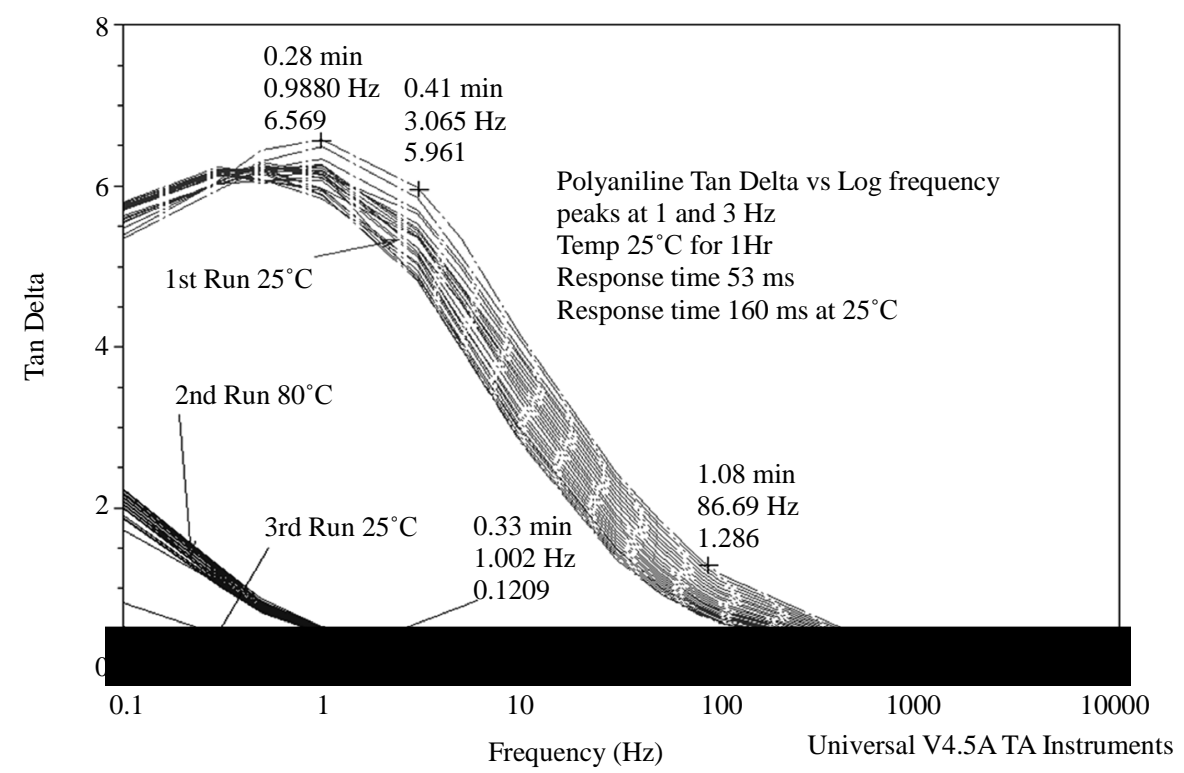

Figure 10. DEA response time analysis for Polyaniline. HCI ERF.

\section{Conclusions}

Evaluation of the physical-chemical and electrical properties of recently synthesized and purchased ERFs revealed that the commercial Fludicon ${ }^{\circledR}$ RheOil 3.0, polyurethane particles coated with Zinc ${ }^{2+}$ ions, was the best candidate in terms of response time (16, 0.16 and $15 \mathrm{~ms}$ ), durability, particle size and settling. Although the Cellulose ERF showed promising response time values (5.5, 0.21 and $1.6 \mathrm{~ms}$ ), larger particle size and fast settling significantly limits the usability in many ERF applications. The PANI. HCl ERF system did not produce characteristics that would allow recommendation as a candidate fluid.

\section{Acknowledgements}

We would like to acknowledge the Third Frontier State of Ohio Research Program (2008-2010), the Office of Sponsored Programs and Research and the College of Science, Cleveland State University for their support and funding.

\section{References}

[1] J. Wei, L. Zhao, S. Peng, J. Shi, Z. Liu and W. Wen, "Wettability of Urea-Doped $\mathrm{TiO}_{2}$ Nanoparticles and Their High Electrorheological Effects," Journal of Sol-Gel Science and Technology, Vol. 47, No. 3, 2008, pp. 311-315. doi:10.1007/s10971-008-1787-z

[2] T. Tilki, M. Yavuz, C. Karabacak, M. Cabuk and M. Ulutuerk, "Investigation of Electrorheological Properties of Biodegradable Modified Cellulose/Corn Oil Suspen- sions," Carbohydrate Research, Vol. 345, No. 5, 2010, pp. 672-679. doi:10.1016/j.carres.2009.12.025

[3] D. P. Park, J. Y. Hwang, H. J. Choi, C. A. Kim and M. S. Jhon, "Synthesis and Characterization of Polysaccharide Phosphates Based Electrorheological Fluids,” Materials Research Innovations, Vol. 7, No. 3, 2003, pp. 161-166. doi:10.1007/s10019-003-0242-6

[4] J. Yin, X. Zhao, X. Xia, L. Xiang and Y. Qiao, "Electrorheological Fluids Based on Nano-Fibrous Polyaniline,” Polymer, Vol. 49, No. 20, 2008, pp. 4413-4419. doi:10.1016/j.polymer.2008.08.009

[5] Y. Liu and P. P. Phule, "Structure Formation in Novel Electrorheological (ER) Fluids Based on Ultrafine Particles of Electronic Ceramics," Polymer Preprints (American Chemical Society, Division of Polymer Chemistry), Vol. 35, No. 2, 1994, pp. 347-348.

[6] D. Kittipoomwong, D. J. Klingenberg, Y. M. Shkel, J. F. Morris and J. C. Ulicny. "Transient Behavior of Electrorheological Fluids in Shear Flow," Journal of Rheology, Vol. 52, No. 1, 2008, pp. 225-241. doi:10.1122/1.2794803

[7] W. M. Winslow, "Induced Fibrillation of Suspensions," Journal of Applied Physics, Vol. 20, No. 12, 1949, pp. 1137-1140. doi:10.1063/1.1698285

[8] Z. P. Shulman, R. Gorodkin, E. Korobko and V. Gleb. "The Electrorheological Effect and Its Possible Uses," Journal of Non-Newtonian Fluid Mechanics, Vol. 8, No. 1-2, 1981, pp. 29-41. doi:10.1016/0377-0257(81)80003-1

[9] J. P. Coulter, K. D. Weiss and J. D. Carlson, "Engineering Applications of Electrorheological Materials,” Journal of Intelligent Material Systems and Structures, Vol. 4, No. 2, 1993, pp. 248-259. doi:10.1177/1045389X9300400215

[10] J. W. Pialet and K. O. Havelka. "Electrorheological Technology: The Future is Now,” Chemtech, Vol. 26, 1996, 
pp. 3645-3653.

[11] C. F. Zukoski, "Material Properties and the Electrorheological Response," Annual Review of Materials Science, Vol. 23, 1993, pp. 45-78. doi:10.1146/annurev.ms.23.080193.000401

[12] A. T. Riga and L. Judovits, "Material Characterization by Dynamic and Modulated Thermal Analytical Techniques,” ASTM Special Technical Publication, USA, 2001.

[13] L. Nunez-Regueira, S. Gomez-Barreiro and C. A. Gracia-Fernandez, "Study of the Influence of Isomerism on the Curing Properties of the Epoxy System DGEBA ( $\mathrm{n}=$ 0)/1,2 DCH by DEA and MDSC," Journal of Thermal Analysis and Calorimetry, Vol. 82, No. 3, 2005, pp. 797-801. doi:10.1007/s10973-005-0966-1

[14] C. M. Kinart, W. J. Kinart, D. Checinska-Majak and A. Bald, "Densities and Relative Permittivities for Mixtures of 2-Methoxyethanol with DEA and TEA, at Various Temperatures," Journal of Thermal Analysis and Calorimetry, Vol. 75, No. 1, 2004, pp. 347-354. doi:10.1023/B:JTAN.0000017355.26845.a4

[15] N. Zanati, M. E. Mathews, N. I. Perera, J. J. Moran, J. A. Boutros, A. T. Riga and M. Bayachou, "Cholesterol Levels and Activity of Membrane Bound Proteins: Characterization by Thermal and Electrochemical Methods,” Journal of Thermal Analysis and Calorimetry, Vol. 96, No. 3, 2009, pp. 669-672. doi:10.1007/s10973-009-0032-5
[16] M. E. Matthews, I. Atkinson, L. Presswala, O. Najjar, N. Gerhardstein, R. Wei, E. Rye and A. T. Riga, "Dielectric Classification of $D$ and $L$ Amino Acids by Thermal and Analytical Methods," Journal of Thermal Analysis and Calorimetry, Vol. 93, No. 1, 2008, pp. 281-287. doi:10.1007/s10973-007-8835-8

[17] L. Johnston, “ Electrorheological Dampers for Industrial and Mobile Applications-An Overview of Design Variations, Product Realisation and Performance,” Proceedings of the 11th International Conference on New Actuators, Bremen, 9-11 June 2008, p. 499.

[18] S. Schneider, K. Holzmann, W. Kemmetmüller, L. Johnston and D. Lißman, "Development and Testing of a Semi-Active Suspension System for Off-Road Trucks Using Electrorheological Dampers," Proceedings of the 11th International Conference on New Actuators, Bremen, 9-11 June 2008, p. 485.

[19] M. Gurka, D. Adams, L. Johnston and R. Petricevic, "New Electrorheological Fluids-Characteristics and Implementation in Industrial and Mobile Applications," Journal of Physics: Conference Series, Vol. 149, No. 1 2009, Article ID 012008.

[20] S. Ulrich, G. Böhme and R. Bruns. "Measuring the Response Time and Static Rheological Properties of Electrorheological Fluids with Regard to the Design of Valves and Their Controllers," Journal of Physics: Conference Series, Vol. 149, No. 1, 2009, Article ID 012031. 